\title{
Starkregenereignisse und ihre Auswirkungen auf das Einsatzgeschehen der Feuerwehr - Das Fallbeispiel Wuppertal
}

\author{
Valerie Wischott ${ }^{1} \cdot$ Michael Leuchner ${ }^{1}$ \\ Eingegangen: 12. Juli 2021 / Überarbeitet: 1. Dezember 2021 / Angenommen: 2. Dezember 2021 / Online publiziert: 5. Januar 2022 \\ (c) Der/die Autor(en) 2021
}

\section{Zusammenfassung}

Starkregen ist schwer vorhersagbar und geht deshalb oft mit schwerwiegenden Folgen in den Siedlungsräumen einher. Die Bewältigung von Schadenslagen erfolgt durch die Feuerwehr als eine der tätigen Organisationen im Bevölkerungsschutz, die bei solchen Ereignissen einer großen Einsatzbelastung ausgesetzt ist. Dieser Beitrag befasst sich mit der Frage, welche Auswirkungen Starkregenereignisse auf das Einsatzgeschehen der Feuerwehr Wuppertal haben und welche meteorologischen und nichtmeteorologischen Einflussfaktoren sich auf dieses auswirken. Dies wurde anhand von kartografischen Raum- und einfachen Korrelationsanalysen sowie 2 Befragungen für das Fallstudiengebiet untersucht. Es konnte ein $\mathrm{Zu}$ sammenhang zu Versiegelungsgrad, Bevölkerungszahl und Topografie gefunden werden. Zu meteorologischen Faktoren wie Niederschlagshöhe konnte kein eindeutiger Zusammenhang identifiziert werden. Die Ergebnisse deuten auf ein Zusammenwirken unterschiedlicher Einflussfaktoren hin. Dies soll zum Anlass genommen werden, den Einfluss relevanter Faktoren weiterführend zu untersuchen sowie die gewonnenen Erkenntnisse in den praktischen Umgang mit der Starkregenthematik zu integrieren.

Schlüsselwörter Bevölkerungsschutz $\cdot$ Klimaanpassung $\cdot$ Sturzflut $\cdot$ Überschwemmung $\cdot$ Klimawandelfolgen

\begin{abstract}
Heavy rainfall is difficult to predict and thus often has serious consequences in populated areas. The fire brigade, as one of the active organizations in civil protection, is responsible for dealing with any damage and is exposed to a large operational load during such events. This article deals with the effects of heavy rain events on the operations of the Wuppertal fire brigade and the impact of meteorological and non-meteorological factors on them. This was investigated by using cartographic, spatial and simple correlation analyses as well as surveys for the case study area. A correlation existed with degree of sealing, population size, and topography. No clear correlation with meteorological factors, e.g., precipitation amount, could be found. The results indicate an interaction of different influencing factors. This should be taken as an opportunity to further investigate the influence of relevant factors and to integrate the knowledge gained into practical handling of the issue of heavy rain.
\end{abstract}

Keywords Civil protection $\cdot$ Climate adaptation $\cdot$ Flash flood $\cdot$ Flooding $\cdot$ Impact of climate change

Michael Leuchner

michael.leuchner@geo.rwth-aachen.de

1 Lehr- und Forschungsgebiet Physische Geographie und Klimatologie, Geographisches Institut, RWTH Aachen University, Wüllnerstr. 5b, 52062 Aachen, Deutschland

\section{Einleitung}

Die Starkregenereignisse 2021 haben eindrücklich vermittelt, dass ein verändertes Klima bereits jetzt in Deutschland spürbar ist und Handlungsbedarf besteht, sich an die zunehmende Häufigkeit von Extremereignissen anzupassen. Dabei stellt Starkregen aufgrund seiner schweren Vorhersagbarkeit ein meteorologisch schwer zu greifendes Phänomen dar (DWD o.J.). Ursächlich für die entstehenden Schäden, die Starkregen in Siedlungsräumen verursacht, sind hauptsächlich die mit ihm einhergehenden sekundären Prozesse 
Abb. 1 Die Stadt Wuppertal wird von der Wupper sowie der sie begleitenden Schwebebahn durchquert und zeichnet sich durch steile Hanglagen entlang des Flusses aus. (Bildquelle: Udo Stärkelings-Pixabay)

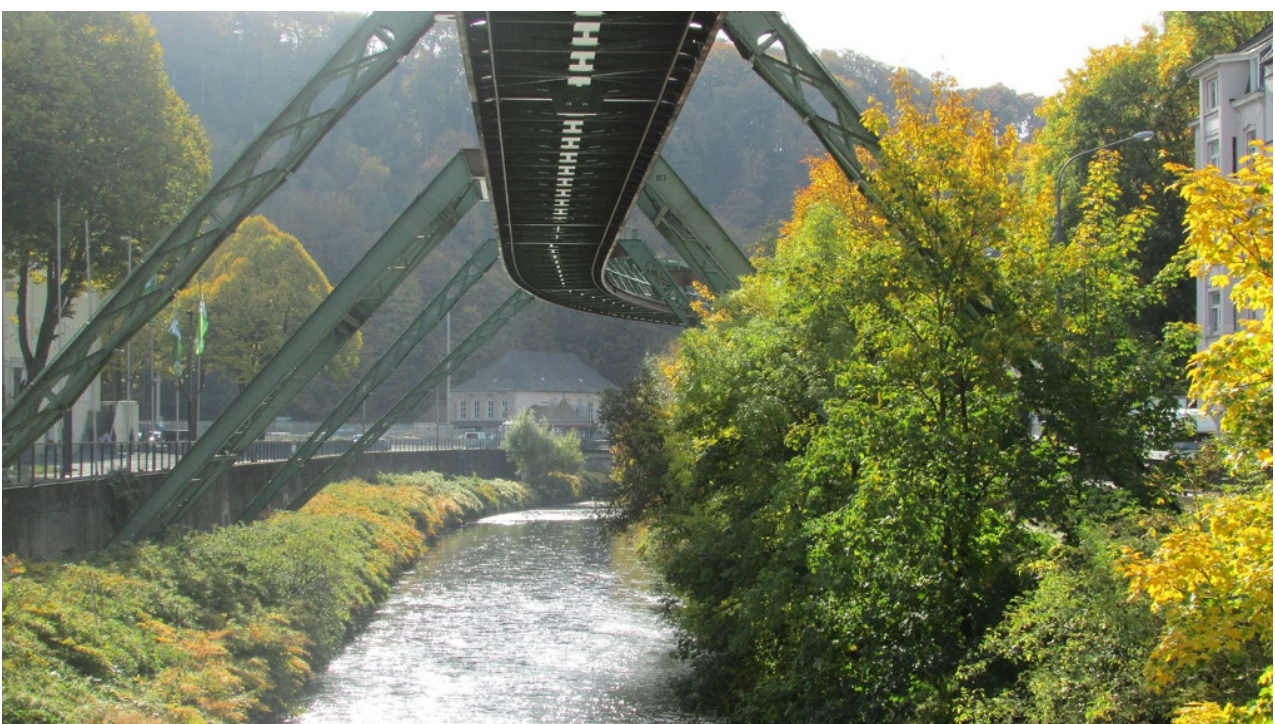

wie die Entstehung von Sturzfluten (Goderbauer-Marchner et al. 2015). Diese oberirdischen Wassermassen bilden sich schlagartig, wenn das Wasser nicht zügig genug versickern oder über die Kanalisation abgeführt werden kann (BBK o.J.). Das Eintreffen von Starkregen ist daher oftmals mit schwerwiegenden und unvorhersehbaren Folgen verbunden. Im Zuge der globalen Erwärmung ist mit intensiveren Starkregen und somit auch einer Zunahme der Häufigkeit und Stärke von Sturzfluten zu rechnen (Westra et al. 2014).

Die Feuerwehr übernimmt bei der Bewältigung eines Starkregenereignisses als im Bevölkerungsschutz tätige Organisation die Aufgabe, Schäden schnellstmöglich zu beseitigen und den vorher bestehenden Zustand wiederherzustellen (Kutschker und Glade 2016). Starkregenereignisse und die damit verbundenen Folgen wie ein stark steigendes und räumlich konzentriertes Notrufaufkommen (Kox und Lüder 2021) tragen zu einer entsprechend großen Einsatzbelastung bei. In der jüngsten Vergangenheit wurden bereits steigende Einsatzzahlen infolge wetterbedingter Einsätze seitens der Feuerwehr signalisiert (Van Rüth et al. 2019).

Die bereits jetzt wahrgenommene hohe Einsatzbelastung durch Starkregenereignisse verbunden mit den globalen Herausforderungen des Klimawandels, erfordern die Entwicklung und Realisierung von Anpassungsmaßnahmen im Bevölkerungsschutz, um die Folgen auch in Zukunft bewältigen zu können. Das seit 2011 laufende Projekt WEXICOM widmet sich z. B. der Optimierung von Warnungen verbunden mit der Frage nach einer adressat*innengerechten Kommunikation von Risiken (Kox und Gerhold 2019). Es muss ein grundlegendes Verständnis über den Zusammenhang zwischen Starkregenereignissen und dem Einsatzgeschehen der Feuerwehr geschaffen werden, um im Kontext der Klimaanpassung die Weiterentwicklung dieser Maßnahmen zu befördern.
In dem Beitrag soll dieser Zusammenhang kritisch betrachtet werden. Dabei wird das Eintreten des Starkregens bis hin zum Einsatzgeschehen als Wirkungskette verstanden, in der die meteorologischen Prozesse, die Charakteristika der davon betroffenen Siedlungsräume sowie der Bevölkerungsschutz zentrale Elemente darstellen. Viele Forschungsprojekte fokussieren die Starkregenthematik auf eine dieser 3 Perspektiven (Nikogosian und Winterrath 2019). Dieser Beitrag liefert einen Ansatz, die Thematik unter $\mathrm{Zu}$ sammenführung der 3 Themenaspekte aus einer interdisziplinären Sichtweise zu betrachten.

Der Zusammenhang zwischen Starkregenereignissen und dem Einsatzgeschehen wird anhand der exemplarischen Betrachtung des Fallstudiengebiets Wuppertal untersucht. Wuppertal stellt aufgrund seiner topografischen Gegebenheiten (Abb. 1) sowie der starken Betroffenheit durch ein markantes Starkregenereignis am 29.05.2018 einen für das Forschungsinteresse geeigneten Raum dar. Es soll untersucht werden, welche räumlichen Auswirkungen Starkregenereignisse auf das Einsatzgeschehen der Feuerwehr Wuppertal hatten und welche meteorologischen und nichtmeteorologischen Einflussfaktoren sich dabei auf das Einsatzgeschehen auswirkten. Die herausgearbeiteten Auswirkungen sollen in einem zusammenführenden Schritt anhand der wirkenden Einflussfaktoren erklärt werden.

\section{Methodischer Ansatz}

Die Analyse umfasste vielfältige Datenquellen, um möglichst diverse Einflüsse auf den Zusammenhang zu untersuchen. Die Basis des Materials bildeten Einsatzdaten der Feuerwehr Wuppertal, die in Form geographischer Koordinaten und unter Angabe des Datums und der Uhrzeit 
Tab. 1 Parameter aus der Starkregenereignisdatenbank, nach denen Starkregenereignisse klassifiziert werden. (angelehnt an Lengfeld et al. 2021)

\begin{tabular}{|c|c|c|}
\hline Parameter & Einheit & Bedeutung \\
\hline Dauer & $\mathrm{h}$ & Signifikante Dauerstufe des Ereignisses \\
\hline Fläche & $\mathrm{km}^{2}$ & Fläche des Ereignisses (Anzahl der RADKLIM-Pixel) \\
\hline RRmean & $\mathrm{mm}$ & Mittlerer Niederschlag: Mittelwert aller Niederschlagshöhen innerhalb der Ereignisfläche \\
\hline Tmean & a & Mittlere Wiederkehrzeit: Mittelwert aller Wiederkehrzeiten innerhalb der Ereignisfläche \\
\hline HPImean & - & $\begin{array}{l}\text { Mittlerer Starkregenindex HPI: Der mittlere HPI beschreibt das Verhältnis der mittleren logarithmischen Wie- } \\
\text { derkehrzeit zur 100-Jährlichkeit (Wertebereich }[0,1] \text { ) }\end{array}$ \\
\hline SRImean & - & $\begin{array}{l}\text { Mittlerer Starkregenindex nach Schmitt (Schmitt et al. 2018): Mittelwert des SRI innerhalb der Ereignisfläche } \\
\text { (Wertebereich }[0,12] \text { ) }\end{array}$ \\
\hline Eta & - & Extremität: Maß, das sowohl die Wiederkehrzeit $(\mathrm{T})$ als auch die Fläche (A) eines Ereignisses betrachtet \\
\hline RRmax & $\mathrm{mm}$ & Maximale Niederschlagshöhe innerhalb der Ereignisfläche \\
\hline Tmax & a & Maximale Wiederkehrzeit innerhalb der Ereignisfläche \\
\hline HPImax & - & Maximaler Starkregenindex HPI: Maximalwert des HPI innerhalb der Ereignisfläche (Wertebereich $[0,1]$ ) \\
\hline SRImax & - & $\begin{array}{l}\text { Maximaler Starkregenindex nach Schmitt (Schmitt et al. 2018): Maximalwert des SRI innerhalb der Ereignis- } \\
\text { fläche (Wertebereich }[0,12] \text { ) }\end{array}$ \\
\hline
\end{tabular}

Tab. 2 Niederschlagsschwellwerte zur Definition eines Objektes, abhängig von Dauerstufe und Mindestfläche. (angelehnt an BBK und DWD 2020)

\begin{tabular}{llllllllllll}
\hline Dauerstufe $(\mathrm{h})$ & 1 & 2 & 3 & 4 & 6 & 9 & 12 & 18 & 24 & 48 & 72 \\
\hline Min. Fläche $\left(\mathrm{km}^{2}\right)$ & 9 & 9 & 9 & 12 & 18 & 27 & 36 & 54 & 72 & 144 & 216 \\
Statistischer Nie- & 13,6 & 17,6 & 20,5 & 22,4 & 25,2 & 28,4 & 30,8 & 34,2 & 36,9 & 44,0 & 48,8 \\
derschlag (mm) & bis & bis & bis & bis & bis & bis & bis & bis & bis & bis & bis \\
nach RADKLIM & 36,7 & 39,7 & 46,9 & 53,0 & 62,7 & 74,3 & 83,7 & 99,2 & 111,8 & 150,1 & 180,0 \\
$\geq 5$ a & & & & & & & & & & &
\end{tabular}

vorlagen und für den Zeitraum 2007-2018 in die Analyse einflossen. Diese wurden mit einer meteorologischen und einer nichtmeteorologischen Datengrundlage rechnerisch und, sofern möglich, grafisch verknüpft, um mögliche Zusammenhänge aufzuzeigen.

Die meteorologische Datengrundlage bildete die im Projekt KlamEx entwickelte Datenbank, in der Starkregenereignisse definiert und klassifiziert wurden (Tab. 1; Lengfeld et al. 2021). Sie basiert auf der Radarklimatologie $R A D$ KLIM des Deutschen Wetterdienstes (DWD), ein ,räumlich und zeitlich hoch aufgelöster Datensatz auf der Basis der radarbasierten Niederschlagsanalysen des DWD“ (BBK und DWD 2020). Die Veröffentlichung der Datenbank erfolgte nach Beendigung dieser Untersuchung, sodass mit einer vorläufigen Version gearbeitet wurde.

Zusätzlich ist jedes Ereignis durch eine georeferenzierte Polygonfläche definiert. Die Definition von Ereignissen in der Datenbank ermöglicht deren genaue raumzeitliche Abgrenzung und stützt sich auf einen Ansatz, der die klimatologische Auftrittswahrscheinlichkeit berücksichtigt und nach statistischen Niederschlagsschwellwerten für verschiedene Wiederkehrzeiten (hier 5 Jahre) selektiert. Der Schwellwert gilt abhängig von verschiedenen Dauerstufen und Flächengrößen des Niederschlags (Tab. 2) (BBK und DWD 2020).

Die Datenbank wurde verwendet, um vergangene Starkregenereignisse in Wuppertal zu identifizieren und den entsprechenden Einsätzen zuzuordnen. Alle Ereignisse, die Einsätze zur Folge hatten, wurden in die Analyse miteinbezogen. Die Datenbank bildete zudem die Basis dafür, Zusammenhänge zwischen ereignisbezogenen Parametern (z.B. Niederschlagsmenge, Ereignisdauer) und der Anzahl an Einsätzen zu untersuchen.

Die nichtmeteorologische Grundlage bildeten insbesondere Geodaten. Die Auswahl orientierte sich u. a. an den von Ahlhelm et al. (2016), Goderbauer-Marchner et al. (2015) und HLNUG (2020) beschriebenen Einflussfaktoren sowie der Datenverfügbarkeit. Zu den untersuchten Geodaten gehörten einerseits die topografischen Faktoren Geländehöhe und Hangneigung, die in Form eines Digitalen Geländemodells integriert wurden. Aus siedlungsbezogener Perspektive wurden die Bevölkerungsdichte und der Versiegelungsgrad analysiert. Tab. 3 stellt eine Übersicht der untersuchten Einflussfaktoren dar.

Ein Schwerpunkt des methodischen Vorgehens lag auf kartografischen Raumanalysen, um räumliche Zusammenhänge aufzuzeigen. Hierfür wurden die verschiedenen Materialien mittels ArcGIS Pro übereinandergelegt (Kutschker 2018) und einfache Korrelationen nach Pearson mit SPSS berechnet, um den Zusammenhang zwischen dem Einsatzaufkommen und den Einflussfaktoren zu quantifizieren. Hochsignifikante Zusammenhänge $(p<0,01)$ wurden mit ** markiert, signifikante $(p<0,05)$ mit *. Die Anzahl der Einsätze wurde jeweils mit den Parametern der Starkre- 
Tab. $3 \mathrm{Zu}$ untersuchende nichtmeteorologische Einflussfaktoren in Form von Geodaten

\begin{tabular}{|c|c|c|c|}
\hline Einflussfaktor & Datenprodukt & $\begin{array}{l}\text { Räumliche Auflö- } \\
\text { sung }\end{array}$ & Datenquelle \\
\hline Bevölkerungszahl & Zensus 2011 & $1000 \mathrm{~m}$ & $\begin{array}{l}\text { https://www.opengeodata.nrw.de/produkte/bevoelkerung/ } \\
\text { zensus2011/ergebnisse_1km-gitter/ }\end{array}$ \\
\hline Versiegelungsgrad & Imperviousness Density 2015 & $20 \mathrm{~m}$ & $\begin{array}{l}\text { https://land.copernicus.eu/pan-european/high-resolution-layers, } \\
\text { imperviousness/status-maps/2015 }\end{array}$ \\
\hline Geländehöhe & SRTM 1 Arc-Second Global & $30 \mathrm{~m}$ & https://earthexplorer.usgs.gov/ \\
\hline Hangneigung & $\begin{array}{l}\text { Ableitung aus SRTM } 1 \text { Arc- } \\
\text { Second Global }\end{array}$ & $30 \mathrm{~m}$ & https://earthexplorer.usgs.gov/ \\
\hline
\end{tabular}

genereignisdatenbank und den 4 verschiedenen Geodatensätzen korreliert.

Eine Erweiterung der Methodik stellen 2 Befragungen von Mitarbeitenden der Feuerwehren Aachen und Wuppertal dar. Die Befragten werden zur Wahrung ihrer Anonymität als B1 (Aachen, mündlich durchgeführt) und B2 (Wuppertal, schriftlich durchgeführt) bezeichnet. Das Ziel lag darin, die grafisch und rechnerisch analysierten Daten durch praxisnahe Perspektiven und Erfahrungen zu ergänzen und zusätzliche Erkenntnisse zu Einflussfaktoren zu gewinnen, die nicht aus räumlichen Daten ableitbar sind bzw. zu denen keine räumlichen Daten vorlagen. Die Aussagen der Feuerwehr Aachen wurden nicht verwendet, um Schlüsse auf Wuppertal zu ziehen.

\section{Auswirkungen von Starkregen auf das Einsatzgeschehen der Feuerwehr}

Insgesamt fanden im Untersuchungszeitraum (2007-2018) im Stadtgebiet Wuppertal 10 Starkregenereignisse an 8 Tagen statt, die eine Gesamtzahl von 1176 Feuerwehreinsätzen zur Folge hatten. In Abb. 2 ist das jeweilige Einsatzaufkommen sowie der räumliche Deckungsgrad zwischen der Ausdehnung der Ereignisse und der Einsatzverteilung dargestellt. Auffällig ist das unterschiedlich hohe Einsatzaufkommen infolge der jeweiligen Ereignisse. Einige Ereignisse hatten niedrige Einsatzzahlen (z. B. 09.08.2007: 39), andere deutlich höhere (z.B. 10.06.2018: 106) zur Folge. Insbesondere sticht der 29.05.2018 mit einem Einsatzaufkommen von 845 heraus. Auf räumlicher Ebene zeigt sich eine grobe Orientierung der Einsatzverteilung an den Starkregenereignisflächen. So umfasste z.B. das Ereignis vom 09.08.2007 in seiner Ausdehnung das komplette Stadtgebiet, während auch die zugehörigen Einsätze breit über die Stadt verteilt waren. Dies gilt ebenso für das eher kleinräumige Ereignis am 10.06.2018. Mit den Ereignissen am 01.06.2018 zeigte sich jedoch auch ein abweichendes Beispiel, bei dem einige Einsätze außerhalb der Starkregenereignisfläche lagen.

Ein detaillierterer Blick auf das markante Ereignis am 29.05.2018 verdeutlicht, wie sich die zahlreichen doku- mentierten Schäden im Stadtgebiet (Westdeutsche Zeitung 2018) im Sinne der eingangs erwähnten Wirkungskette in einem entsprechend hohen Einsatzaufkommen widerspiegeln. Das aus nordöstlicher Richtung anströmende Ereignis mit einer mittleren Niederschlagshöhe von $41,8 \mathrm{~mm}$ dauerte 2 Stunden (12:50-14:50 Uhr UTC) und überdeckte eine Fläche von $71 \mathrm{~km}^{2}$ über dem nördlichen Wuppertal. Die räumliche Verteilung der Einsatzschwerpunkte konzentrierte sich auf den südlichen Teil der Starkregenereignisfläche und dort schwerpunktmäßig in den Innenstädten Barmen und Elberfeld (B2) sowie um die Bereiche der Wupper und entlang von Straßenzügen (Abb. 3).

\section{Einflussfaktoren auf das Einsatzgeschehen der Feuerwehr}

Das Einsatzgeschehen infolge eines Starkregenereignisses wird an erster Stelle durch die dem Starkregen eigenen Eigenschaften beeinflusst: Das Schadensausmaß lässt sich maßgeblich auf die Unvorhersagbarkeit und die Intensität solcher Ereignisse zurückführen (Goderbauer-Marchner et al. 2015). Dennoch liefert die räumliche Darstellung der 10 Ereignisse und der Verteilung der Einsätze (Abb. 2) einen ersten Hinweis darauf, dass der Ort der Schadensentstehung und damit des Einsatzgeschehens nicht primär vom Ort des Niederschlagsgeschehens abhängig zu sein scheint, was auf weitere Einflussfaktoren schließen lässt. Die kartografischen und Korrelationsanalysen unter Einbezug der Befragungen deuten an, dass das Zusammenspiel einer Vielzahl an Faktoren dafür verantwortlich ist, wie sich ein Starkregenereignis im Stadtgebiet auswirkt und welches Einsatzaufkommen es nach sich zieht. Die Faktoren, die in der vorliegenden Untersuchung den stärksten Zusammenhang zum Einsatzgeschehen aufwiesen, waren nichtmeteorologischer Natur. Die untersuchten meteorologischen Faktoren zeigten keinen eindeutigen Zusammenhang auf.

Aus nicht-meteorologischer Perspektive ergaben sich aus den Analysen signifikante Zusammenhänge zu den Siedlungsfaktoren Bevölkerungsdichte $\left(r=0,70^{* *}\right)$ und Versiegelungsgrad $\left(r=0,23^{*}\right)$ sowie den topografischen Faktoren Geländehöhe $\left(\mathrm{r}=-0,41^{* *}\right)$ und Hangneigung $\left(\mathrm{r}=-0,15^{* *}\right)$. 
Abb. 2 Starkregenereignisse und Einsatzaufkommen in Wuppertal (2007-2018)

Abb. 3 Einsatzaufkommen in Wuppertal infolge des Starkregenereignisses am 29.05.2018; a Schwerpunkte nördlich der Wupper um den Bereich Alter Markt in Barmen; b Schwerpunkte rund um den Bereich Kirchplatz und nördlich davon entlang der Gathe
Starkregenereignisse und Einsatzaufkommen in Wuppertal (2007-2018)
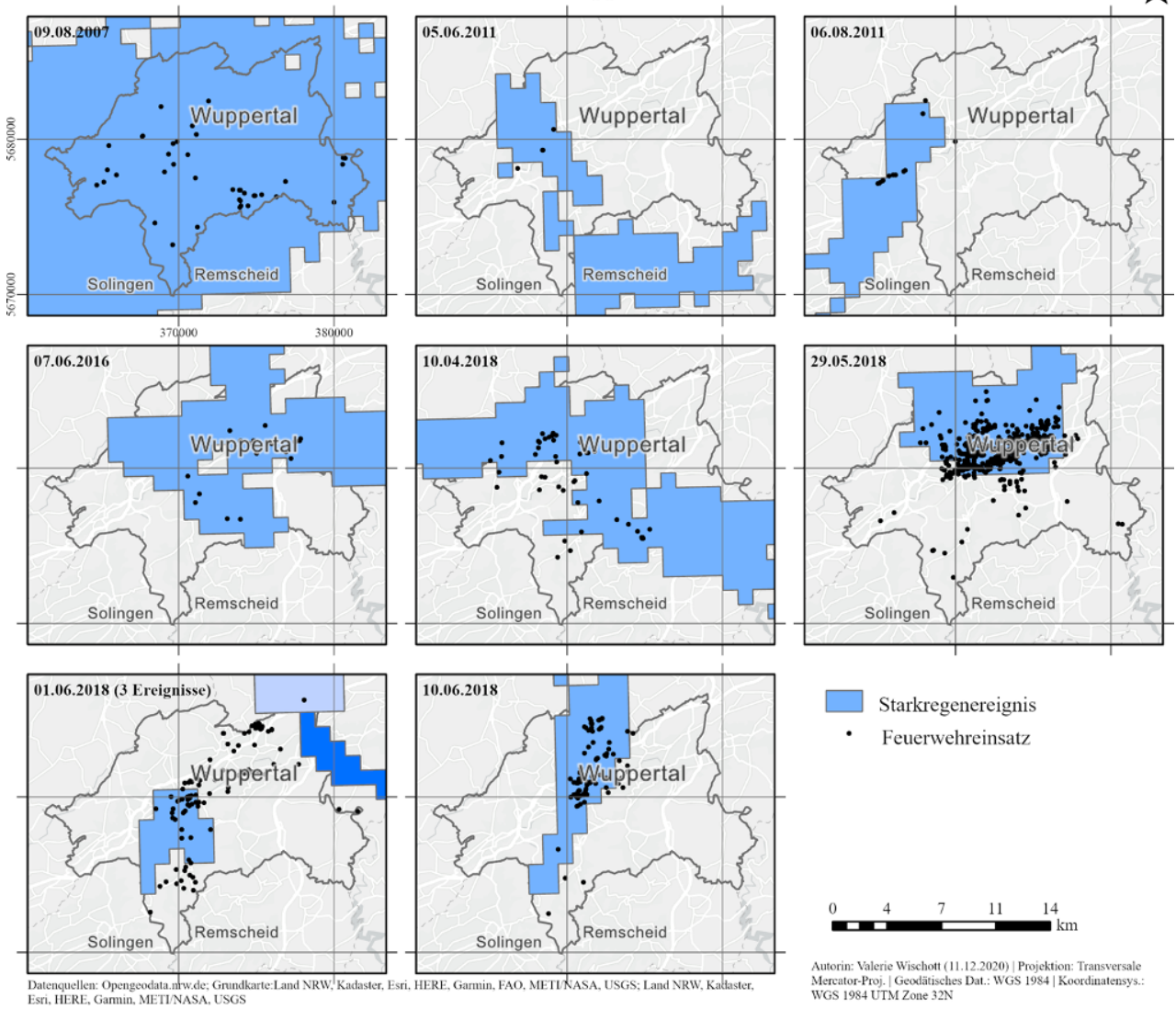

Starkregenereignis

- Feuerwehreinsatz

Starkregenereignis am 29.05.2018 und Einsatzaufkommen in Wuppertal

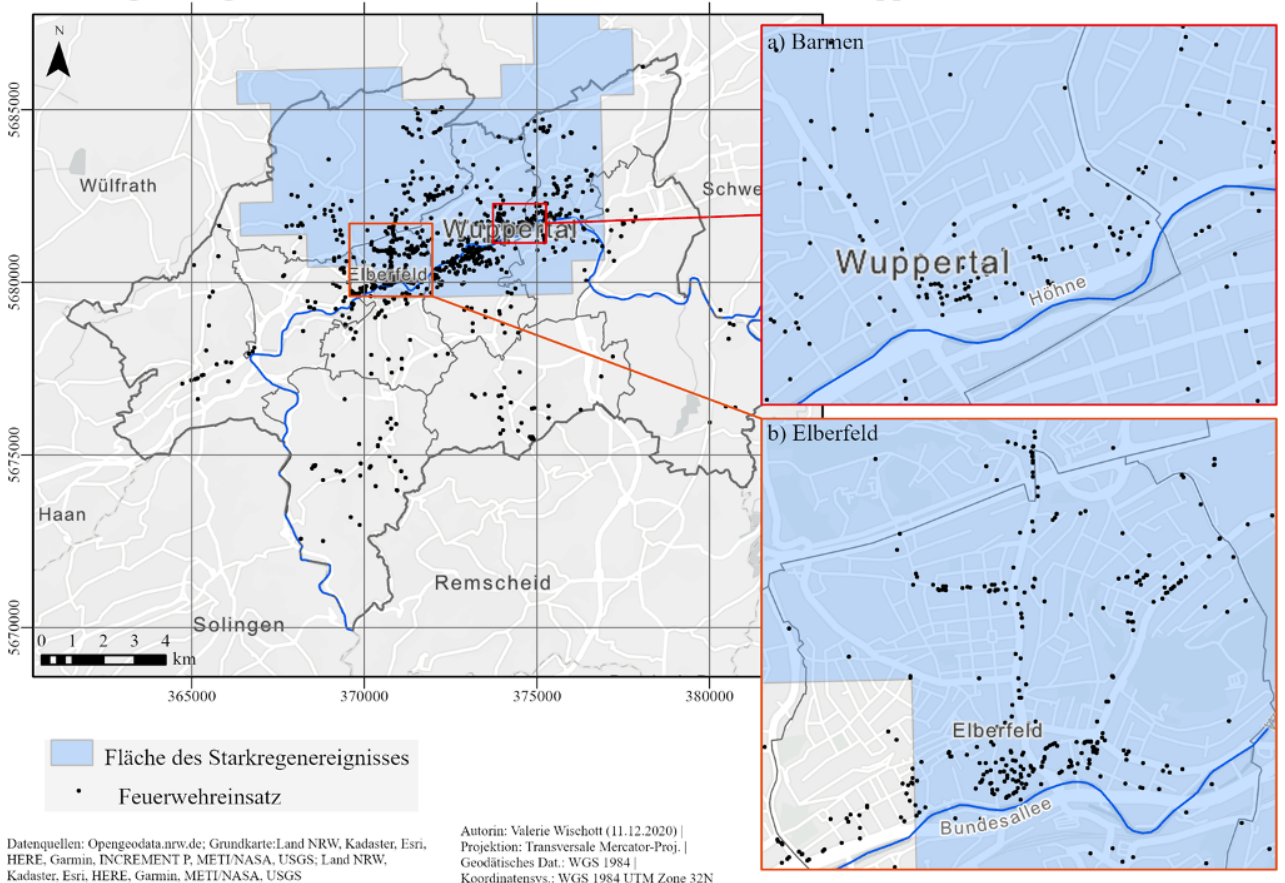


Abb. 4 Geländehöhe und Einsatzaufkommen in Wuppertal; Darstellung aller Einsätze infolge der 10 Starkregenereignisse im Untersuchungszeitraum (2007-2018)

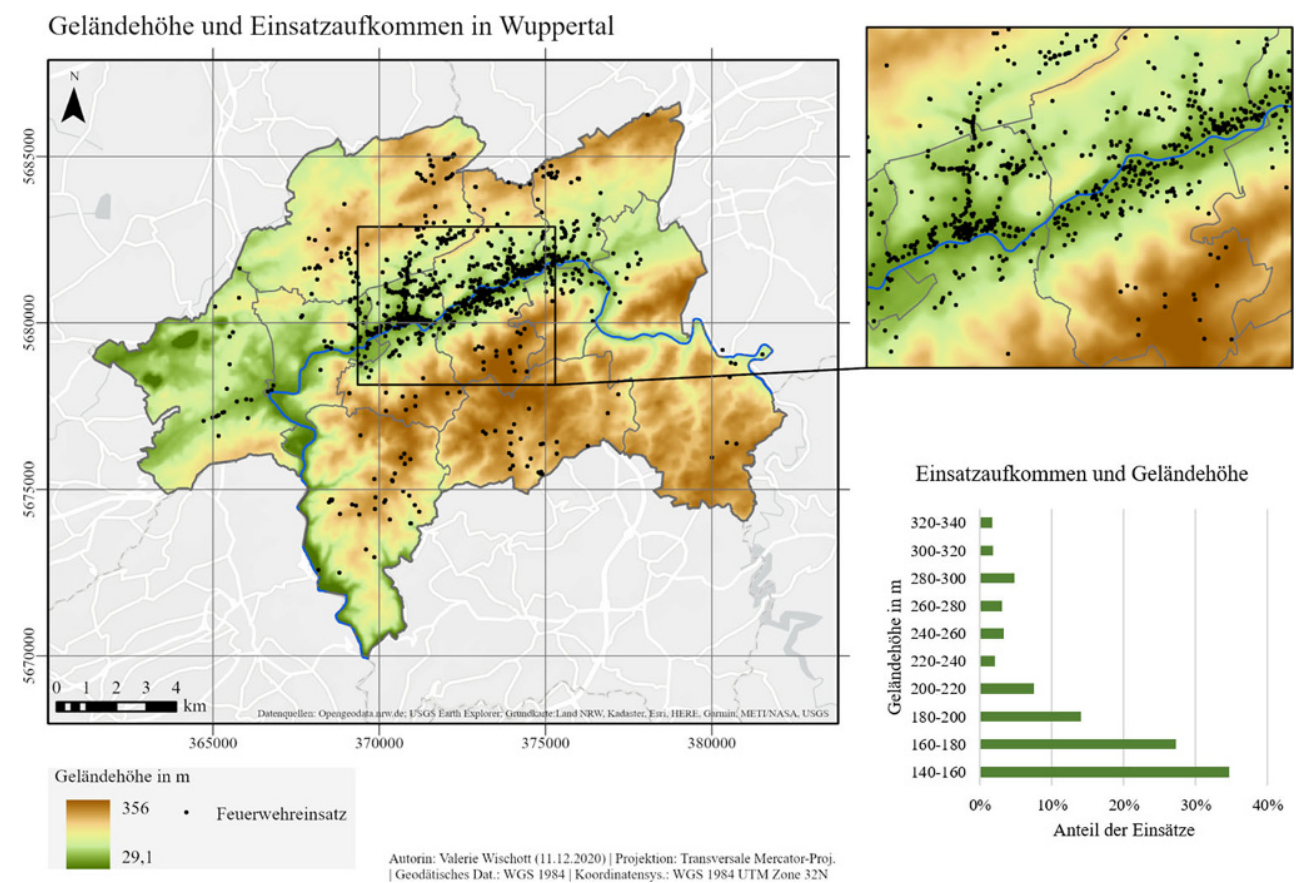

Beispielhaft ist der Zusammenhang zwischen Geländehöhe und Einsatzaufkommen in Abb. 4 dargestellt. Im Zuge der Befragungen wurde zudem angemerkt, dass die Leistungsfähigkeit der Kanalisation sowie die Versickerungsfähigkeit des Bodens, die z.B. nach langanhaltenden Trockenphasen vermindert ist, einen großen Einfluss auf sich bildende Einsatzschwerpunkte haben könnte (B1; B2). In Wuppertal kam zudem der Einfluss der Wupper zum Tragen, deren starke Pegelzunahme innerhalb kürzester Zeit zu einem Rückstau des Wassers führte (B2).

Neben diesen Faktoren wurde aus Perspektive der Feuerwehr zudem das Verhalten der Bevölkerung als Einflussfaktor auf die entstehenden Schäden und damit auf die Einsatzhäufigkeit der Feuerwehr gesehen. Insbesondere die Selbsthilfefähigkeit der Bevölkerung könne eine große Bedeutung für die Verminderung der Schadensentstehung einnehmen. Selbsthilfemaßnahmen, wie z.B. die eigenständige Konstanthaltung des Wasserpegels im Keller durch eine Tauchpumpe (B1), können demnach Einsatzstellen vermeiden und die Bildung von Schwerpunkten beeinflussen (Nikogosian et al. 2021).

Die Wirkung mehrerer nichtmeteorologischer Faktoren an einem Ort kann beispielhaft an den gefundenen Einsatzschwerpunkten am 29.05.2018 veranschaulicht werden (Abb. 3). Diese wurden vor allem in Teilen der Innenstadt festgestellt, welche sich durch ihre Tallage auszeichnet. Die Hangneigung verstärkt den Oberflächenabfluss, welcher sich in den Tälern sammelt und dort Schäden verursacht. Die Innenstadtbereiche weisen auch hohe Bevölkerungsdichten auf, was zu einer großen Anzahl an potenziell von Schäden betroffenen Personen führen kann. Die zu- sätzlich stark ausgeprägte Bodenversiegelung führt zu einem verstärkten Oberflächenabfluss und zur Überlastung der Kanalisation (Kutschker 2018). Die Wupper kann als zusätzlicher Einflussfaktor für das erhöhte Einsatzaufkommen hinzugezogen werden.

Daraus folgt, dass die Anzahl und Lokalisierung der Einsätze nach einem Starkregen maßgeblich davon abhängen, welche und wie viele der nichtmeteorologischen Faktoren sich in einem Gebiet vereinen. Dies begründet auch, warum das Einsatzaufkommen nach Ereignissen generell sehr unterschiedlich ausfallen kann (Abb. 2).

Zudem kann vermutet werden, dass die Vorgehensweise der Feuerwehren einen Einfluss auf die Bildung von Einsatzschwerpunkten haben kann. Nach Eintritt eines Starkregens verschafft sich die Feuerwehr zunächst einen Überblick über die Gesamtlage sowie über Schadensschwerpunkte. Die Abarbeitung der Notrufe erfolgt dann nach Priorisierung; so hat die Menschenrettung Vorrang vor Sachwertschutz und die Sicherstellung der Verfügbarkeit Kritischer Infrastrukturen vor individuellem Besitz (B2; Nikogosian et al. 2021). Diese ,abwartende“ (Nikogosian et al. 2021) Taktik könnte sich auf die raumzeitliche Entwicklung von Einsatzstellen auswirken.

Die ausschlaggebende Wirkung der untersuchten nichtmeteorologischen Faktoren auf das Einsatzgeschehen konnte in der vorliegenden Untersuchung festgestellt werden (vgl. auch Nikogosian et al. 2021). Weniger deutlich hingegen zeigte sich der Zusammenhang zu den meteorologischen Faktoren. So ergaben sich für die Wiederkehrzeit $\left(r_{\text {Tmax }}=0,99^{*}, r_{\text {Tmean }}=0,97^{*}\right)$ und den daraus abgeleiteten Heavy Precipitation Index $\left(\mathrm{r}_{\mathrm{HPI} \text { mean }}=0,69 *\right)$ signifikante Er- 
gebnisse. Für die restlichen 9 Parameter ergaben sich keine bedeutsamen Zusammenhänge.

Es scheint, dass das Ausmaß sowie der Ort der Schadensentstehung und damit des Einsatzgeschehens nicht primär von den untersuchten meteorologischen Parametern abhängig waren. Einerseits gibt dies zum Anlass, die Datengrundlage unter Einschluss weiterer Fallstudiengebiete bzw. Einsatzdaten zu vergrößern, um somit solidere statistische Aussagen zu erzielen. Andererseits geben die Ergebnisse Hinweise auf weitere meteorologische Einflüsse, die nicht Bestandteil der untersuchten Ereignisdatenbank waren, wie z.B. die Niederschlagsart. Für den Starkregen am 29.05.2018 in Wuppertal sei insbesondere der starke Hagel zu Beginn ein Auslöser für viele Schäden gewesen, da er Verstopfungen verursachte (B2). Hagel kann nicht nur den Wasserabfluss blockieren, sondern auch erhebliche unmittelbare Schäden verursachen, z. B. an Dächern und Fassaden (GDV 2019). Die Ergebnisse geben zum Anlass, den räumlichen Verlauf des Niederschlagsgeschehens in die Analyse miteinzubeziehen. So könnte, z.B. unter Berücksichtigung der jeweiligen Lage der Gewässereinzugsgebiete, untersucht werden, ob die Zugrichtung des Niederschlags das Einsatzgeschehen in seiner raumzeitlichen Verteilung beeinflusst.

\section{Fazit}

Im Zuge der Untersuchung konnte ein umfassendes Bild der räumlichen Auswirkungen von Starkregen auf das Einsatzgeschehen der Feuerwehr sowie einige der darauf wirkenden Faktoren für Wuppertal erlangt werden. Es wurden Hinweise auf weitere wirkende meteorologische Faktoren aufgedeckt und damit eine Erweiterung zu deren Forschungstätigkeit gegeben. Auch geben die Erkenntnisse über die Einsatzschwerpunkte und die nichtmeteorologischen Faktoren zum Anlass, diese in den praktischen Umgang mit Starkregen zu integrieren. Hier sei die Sensibilisierung und Stärkung der Resilienz der Bevölkerung, insbesondere die Selbsthilfefähigkeit, als eine Stellschraube zur Schadensverminderung und damit Entlastung der Feuerwehr zu nennen. Ebenso geben die Ergebnisse Anlass dazu, Wissensbestände zu integrieren. Dies kann z.B. durch den Abgleich der Einsatzschwerpunkte mit und damit die Validierung von Starkregengefahrenkarten geschehen, deren Entwicklung in Wuppertal bereits Realität ist (Stadt Wuppertal o.J.). Zudem könnten das Monitoring der Pegelstände von Fließgewässern und die Etablierung eines entsprechenden Warnsystems dazu gehören. Gebündelt liefert die Integration dieser Elemente das Potenzial, Maßnahmenkonzepte für die Einsatzplanung der Feuerwehr zu entwickeln. So kann ein Beitrag zur Prävention von und Vorbereitung auf in Zukunft möglicherweise zunehmende Starkregenereignisse und damit zur Klimaanpassung geliefert werden.

Insgesamt muss bei dieser Untersuchung berücksichtigt werden, dass die Ergebnisse eingeschränkt und ausschließlich für den Raum Wuppertal gültig sind. Die Einschränkung bezieht sich auf die Korrelationsberechnungen der Parameter aus der Ereignisdatenbank, die auf einer für statistische Zwecke recht kleinen Stichprobe beruhte. Zum Vergleich der Ergebnisse mit anderen Räumen und der Ableitung übergreifender Aussagen daraus ist eine einheitliche Datenbasis eine Voraussetzung. Empfehlungen zu einer systematischen Erfassung starkregenbedingter Feuerwehreinsätze sowie den daraus entstehenden Mehrwert geben Nikogosian et al. (2021). Trotz dieser Unsicherheiten ist eine Übereinstimmung der Ergebnisse mit anderen im Projekt KlamEx untersuchten Räumen festzustellen (Nikogosian et al. 2021).

Danksagung Das Projekt Klassifikation meteorologischer Extremereignisse zur Risikovorsorge gegenüber Starkregen für den Bevölkerungsschutz und die Stadtentwicklung (KlamEx) wird von den Partnern der Strategischen Behördenallianz Anpassung an den Klimawandel BBK, Bundesinstitut für Bau-, Stadt- und Raumforschung, DWD, Technisches Hilfswerk und Umweltbundesamt durchgeführt. Ein herzlicher Dank geht an das BBK und den DWD für die Unterstützung und Berechtigung zur Verwendung der Daten sowie an die Feuerwehren Wuppertal und Aachen für die Interviews und die Bereitstellung der Einsatzdaten.

Funding Open Access funding enabled and organized by Projekt DEAL.

Open Access Dieser Artikel wird unter der Creative Commons Namensnennung 4.0 International Lizenz veröffentlicht, welche die Nutzung, Vervielfältigung, Bearbeitung, Verbreitung und Wiedergabe in jeglichem Medium und Format erlaubt, sofern Sie den/die ursprünglichen Autor(en) und die Quelle ordnungsgemäß nennen, einen Link zur Creative Commons Lizenz beifügen und angeben, ob Änderungen vorgenommen wurden.

Die in diesem Artikel enthaltenen Bilder und sonstiges Drittmaterial unterliegen ebenfalls der genannten Creative Commons Lizenz, sofern sich aus der Abbildungslegende nichts anderes ergibt. Sofern das betreffende Material nicht unter der genannten Creative Commons Lizenz steht und die betreffende Handlung nicht nach gesetzlichen Vorschriften erlaubt ist, ist für die oben aufgeführten Weiterverwendungen des Materials die Einwilligung des jeweiligen Rechteinhabers einzuholen.

Weitere Details zur Lizenz entnehmen Sie bitte der Lizenzinformation auf http://creativecommons.org/licenses/by/4.0/deed.de.

\section{Literatur}

Ahlhelm I, Frerichs S, Hinzen A, Noky B, Simon A, Riegel C, Trum A, Altenburg A, Janssen G, Rubel C (2016) Klimaanpassung in der räumlichen Planung (Praxishilfe) - Starkregen, Hochwasser, Massenbewegungen, Hitze, Dürre. Umweltbundesamt, DessauRoßlau

BBK (o.J.) Naturgefahr: Starkregen. https://www.bbk.bund.de/DE/ Themen/Risikomanagement/Baulicher-Bevoelkerungsschutz/ 
Schutz-vor-Naturgefahren/Starkregen/starkregen_node.html. Zugegriffen: 9. Okt. 2021

BBK, DWD (Hrsg) (2020) Klassifikation meteorologischer Extremereignisse zur Risikovorsorge gegenüber Starkregen für den Bevölkerungsschutz und die Stadtentwicklung (KlamEx) (Zwischenbericht)

DWD (o.J.) Unwetterklimatologie: Starkregen. https://www.dwd.de/ DE/leistungen/unwetterklima/starkregen/starkregen_node.html. Zugegriffen: 14. Okt. 2021

GDV (2019) Hagelregister. https://www.gdv.de/de/themen/schaedendurch-hagel-51366. Zugegriffen: 14. Okt. 2021

Goderbauer-Marchner G, Sontheimer R, Maier T, Ziegler J, Alte F, Lorenz D, Renz C (2015) Die unterschätzten Risiken „Starkregen“ und „Sturzfluten“ - Ein Handbuch für Bürger und Kommunen. Bundesamt für Bevölkerungsschutz und Katastrophenhilfe, Bonn

HLNUG (2020) Schäden durch Starkregen vermeiden. Hessisches Landesamt für Naturschutz, Umwelt und Geologie, Wiesbaden

Kox T, Gerhold L (2019) Wetterwarnungen: Von der Extremereignisinformation zu Kommunikation und Handlung. Beiträge aus dem Forschungsprojekt WEXICOM. Schriftenreihe Sicherheit, Bd. 25. Forschungsforum Öffentliche Sicherheit, Freie Universität Berlin, Berlin

Kox T, Lüder C (2021) Impacts as triggers for weather-related decision making: observations at the Berlin fire brigade control and dispatch center. Int J Disaster Risk Sci. https://doi.org/10.1007/ s13753-021-00356-4

Kutschker T (2018) Auswirkungen von Starkregenereignissen auf die Gefahrenabwehr im Ballungsraum Rhein-Main und mögliche Anpassungsstrategien im Bevölkerungsschutz. Universität Wien, Wien (Dissertation)

Kutschker T, Glade T (2016) Unwetter! Starkregen als Herausforderung für den anwendungsbezogenen Bevölkerungsschutz. In: Kuhlmey M, Freudenberg D (Hrsg) Krisenmanagement - Bevölkerungsschutz. https://elibrary.duncker-humblot.com/publikation/ b/id/36150/. Duncker\&Humblot, Berlin, S 465-489 https://doi. org/10.13140/RG.2.1.4525.8481
Lengfeld K, Walawender E, Winterrath T, Weigl E, Becker A (2021) Heavy precipitation events Version 2021.01 exceeding return period of 5 years from RADKLIM-RW Version 2017.002. https:// doi.org/10.5676/DWD/CatRaRE_T5_Eta_v2021.01. Zugegriffen: 3. Juli 2021

Nikogosian C, Winterrath T (2019) Extrem gut untersucht? Extremwetter als interdisziplinäres Thema in der deutschen Forschungslandschaft. In: BBK (Hrsg) Klimawandel und Bevölkerungsschutz. Bevölkerungsschutz, Bd. 2. Bundesamt für Bevölkerungsschutz und Katastrophenhilfe, Bonn, S 12-16

Nikogosian C, Winterrath T, Walawander E, Fischer I, SchmitzKröll D, Wischott V (2021) Klassifikation meteorologischer Extremereignisse zur Risikovorsorge gegenüber Starkregen für den Bevölkerungsschutz und die Stadtentwicklung (Projekt der Strategischen Behördenallianz „Anpassung an den Klimawandel“. Abschlussbericht)

Schmitt TG, Krüger M, Pfister A, Becker M, Mudersbach C, Fuchs L, Hoppe H, Lakes I (2018) Einheitliches Konzept zur Bewertung von Starkregenereignissen mittels Starkregenindex. Korrespondenz Abwasser Abfall 65(2):113-120. https://doi.org/10.3242/ kae2018.02.002

Stadt Wuppertal (o.J.) Klimawandel und Starkregen - Starkregengefahrenkarte für Wuppertal. https://www.wuppertal.de/rathausbuergerservice/umweltschutz/immission/starkregen.php. Zugegriffen: 3. Juli 2021

Van Rüth P, Schönthaler K, Von Andrian-Werburg S, Buth M (2019) Monitoringbericht 2019 zur Deutschen Anpassungsstrategie an den Klimawandel. Umweltbundesamt, Dessau-Roßlau

Westdeutsche Zeitung (2018) Unwetter in Wuppertal - Tankstelle bricht zusammen, Bahnverkehr gestört. https://www.wz.de/nrw/ wuppertal/unwetter-in-wuppertal-tankstelle-bricht-zusammenbahnverkehr-gestoert_aid-25222667. Zugegriffen: 3. Juli 2021

Westra S, Fowler HJ, Evans JP, Alexander LV, Berg P, Johnson F, Kendon EJ, Lenderink G, Roberts NM (2014) Future changes to the intensity and frequency of short-duration extreme rainfall. Rev Geophys 52(3):522-555. https://doi.org/10.1002/2014RG000464 